\title{
HDAC2 regulates cell proliferation, cell cycle progression and cell apoptosis in esophageal squamous cell carcinoma EC9706 cells
}

\author{
SHENGLEI LI $^{1 *}$, FENG WANG ${ }^{2 *}$, YUNHUI QU ${ }^{3}$, XIAOQI CHEN ${ }^{2}$, MING GAO $^{2}$, \\ JIANPING YANG ${ }^{1}$, DANDAN ZHANG ${ }^{1}$, NA ZHANG ${ }^{1}$, WENCAI LI ${ }^{1}$ and HONGTAO LIU ${ }^{4}$ \\ Departments of ${ }^{1}$ Pathology and ${ }^{2}$ Oncology; ${ }^{3}$ Clinical Laboratory, The First Affiliated Hospital of Zhengzhou University, \\ Zhengzhou, Henan 450052; ${ }^{4}$ Laboratory for Cell Biology of School of Life Sciences, \\ Zhengzhou University, Zhengzhou, Henan 450001, P.R. China
}

Received June 22, 2015; Accepted October 26, 2016

DOI: $10.3892 / \mathrm{ol} .2016 .5436$

\begin{abstract}
Increasing evidence has demonstrated that histone deacetylase 2 (HDAC2) participates in the regulation of a variety of biological processes in numerous tumors. However, the potential role of HDAC2 in the development and progression of esophageal squamous cell carcinoma (ESCC) remains elusive. Immunohistochemistry was utilized to detect the expression of HDAC2, Cell Counting Kit-8 was used to determine the cell proliferation, and flow cytometry was employed to investigate cell cycle and cell apoptosis. Finally, western blotting was employed to detect the protein expression of cyclin D1, p21, B cell lymphoma-2 (Bcl-2) and Bcl-2-associated $\mathrm{X}$ protein (Bax). The present study found that expression of HDAC2 protein in ESCC tissues was significantly increased compared with atypical hyperplasia tissues and normal esophageal mucosa $(\mathrm{P}<0.001)$. The expression of HDAC2 was not associated with the age or gender of patients $(\mathrm{P}>0.05)$, but was closely associated with the histological grade, invasion depth, tumor-node-metastasis stage and lymph node metastasis, respectively (all $\mathrm{P}<0.001$ ). HDAC2 small interfering RNA effectively downregulated the expression of HDAC2 protein in ESCC EC9706 cells. Downregulation of HDAC2 expression evidently inhibited cell proliferation, arrested cell cycle at the G0/G1 phase and induced cell apoptosis in ESCC EC9706 cells, coupled with increased expression of p21 and
\end{abstract}

Correspondence to: Professor Hongtao Liu, Laboratory for Cell Biology of School of Life Sciences, Zhengzhou University, 100 Kexue Avenue, Zhengzhou, Henan 450001, P.R. China

E-mail: liuht1230@126.com

Professor Wencai Li, Department of Pathology, The First Affiliated Hospital of Zhengzhou University, 40 Daxue Road, Zhengzhou, Henan 450052, P.R. China

E-mail: liwencaipatho@126.com

${ }^{*}$ Contributed equally

Key words: histone deacetylase 2, esophageal squamous cell carcinoma, cell proliferation, cell cycle, cell apoptosis
Bax proteins and decreased expression of cyclin D1 and Bcl-2 proteins. Overall, the present findings suggest that HDAC2 may play an important role in the development and progression of ESCC and be considered as a novel molecular target for the treatment of ESCC.

\section{Introduction}

Esophageal cancer (EC) is a gastrointestinal cancer that is the eighth most common cancer and the sixth most lethal cancer worldwide (1-4). The incidence of EC, particularly esophageal squamous cell carcinoma (ESCC), which is the most prevalent histological type of EC, exhibits a significant geographical distribution, with areas of high incidence located mainly in China, South Asia, East Africa and South Africa $(3,5)$. Currently, the prognosis of patients with ESCC is quite poor, with a 5-year relative survival rate of $<10 \%$ (6). Since early-onset ESCC is asymptomatic, the majority of diagnosed ESCCs are already in a late stage of disease. It is therefore imperative to identify new molecular diagnostic markers for early intervention in ESCC.

Deacetylases are frequently dysregulated in numerous tumors $(7,8)$, Histone deacetylase 2 (HDAC2), an important member of type I histone deacetylases, plays a crucial role in tumor onset and development $(8,9)$. Increasing evidence has revealed that HDAC2 is often overexpressed in various types of tumors (10-15). In addition, the ablation of HDAC2 markedly inhibits tumor growth and induces apoptosis $(13,14,16)$, suggesting HDAC2 functions as oncogene in these tumors. Most importantly, monitoring HDAC2 expression during treatment can act as a marker for the efficacy of HDAC inhibitors (HDACis), and thus the HDAC2 level represents an independent prognostic marker in the clinic $(17,18)$. However, at present, the role of HDAC2 in the development and progression of ESCC remains to be elucidated. Therefore, in the current study, HDAC2 expression in ESCC tissue was determined by immunohistochemistry. EC9706 cells were also transfected with liposome-mediated HDAC2 small interfering (si)RNA, followed by cell proliferation, cell cycle and cell-apoptosis assays, and the underlying molecular mechanism was analyzed. Overall, the present findings indicated that HDAC2 may be a novel molecular target for the therapy of ESCC. 


\section{Materials and methods}

Tissue samples. For the tissue samples, 69 patients with ESCC were enrolled from Anyang Tumor Hospital (Anyang, China), an area with a high incidence of esophageal cancer (19). In total, 45 atypical hyperplasia tissue specimens within $3 \mathrm{~cm}$ of the tumor and another 69 samples of normal tissue were used as controls. Of the 69 patients with esophageal cancer, 41 were male and 28 were female. In total, 27 patients were $\leq 60$ years old, and 42 patients were $>60$ years old. Standards of histological grading were as follows: Grade I, highly differentiated squamous cell carcinoma, with keratin pearls and intercellular bridges; grade II, rare intercellular bridges that are not as evident as in grade I, and the cells of the special type of medium; and grade III, poorly differentiated squamous cell carcinoma, cell dispersion distribution, large and irregular nucleus, and absence of intercelllar bridges. Patients showed the following histology: Grade I, 19 patients; grade II, 27 patients; and grade III, 23 patients. Additionally, 22 patients exhibited shallow invasion to the submucosa or superficial-layer muscle, and 47 patients exhibited deep invasion to deep-layer muscle or fibrous membrane. Based on the Union for International Cancer Control 1997 tumor-node-metastasis (TNM) staging criteria, 33 patients were diagnosed with stage I-II disease and 36 patients were diagnosed with stage III-IV disease. In addition, 31 patients had lymph node metastasis and 38 patients did not. All patients had no history of chemotherapy or radiotherapy. All the aforementioned specimens were fixed in $4 \%$ paraformaldehyde, conventionally dehydrated, embedded in paraffin, serially sectioned (4-6 $\mu \mathrm{m}$ thick), and stained by hematoxylin-eosin (HE) staining (Beijing Zhongshan Golden Bridge Biotechnology Co., Ltd., Beijing, China). The classification of normal, atypical hyperplasia and tumor tissues was confirmed by HE staining. The present study was approved by the Ethical Review Committee of the First Affiliated Hospital of Zhengzhou University (Zhengzhou, China) and written informed consent was obtained from all participants.

Evaluation of immunohistochemistry and staining. Immunohistochemistry was performed as previously described (20). Briefly, streptavidin-peroxidase immunohistochemistry (Beijing Zhongshan Golden Bridge Biotechnology Co., Ltd.) was performed using mouse anti-HDAC2 monoclonal antibody (dilution, 1:200; catalog no., sc-81599; Santa Cruz Biotechnology, Inc., Dallas, TX, USA) at a dilution of 1:200, and 3,3'-diaminobenzidine chromogenic reagent (Beijing Zhongshan Golden Bridge Biotechnology Co., Ltd.) was utilized to develop the staining signal. Hematoxylin was used to counterstain the background, according to the manufacturer's protocol (Yixin Biological Technology, Inc., Shanghai, China). Known HDAC2-positive slices were used as a positive control for each tissue, and PBS was used in place of primary antibody for a negative control. Under high magnification (x400), 5 fields were randomly selected (cell number, $\geq 200$ ), and the percentage of stained cells and depth of staining were used as diagnostic criteria. Specimens were first scored by the percentage of positive cells: 0 for $<5 \%$; 1 for $5-25 \%$; 2 for $25-50 \%$; 3 for $50-75 \%$; and 4 for $\geq 75 \%$. Specimens were then scored by depth of staining: 1 for no or extremely light staining; 2 for light yellow staining; 3 for moderate yellow staining; and 4 for brown staining. HDAC2 protein expression was determined by multiplication of the positive cell number score and the depth of staining score, and was considered to indicate no expression if $<3$ and to indicate expression if $\geq 3$.

Cell culture and transfection. EC9706 cells (American Type Culture Collection, Manassas, VA, USA) were cultured in RPMI-1640 medium (Gibco; Thermo Fisher Scientific, Inc., Waltham, MA, USA) supplemented with $10 \%$ fetal bovine serum (Hangzhou Evergreen Biological Engineering Materials Co., Ltd., Hangzhou, China), $100 \mathrm{U} / \mathrm{ml}$ penicillin and $100 \mu \mathrm{g} / \mathrm{ml}$ streptomycin at $37^{\circ} \mathrm{C}$ in a humidified atmosphere containing $5 \% \mathrm{CO}_{2}$. When cell confluency reached $\sim 90 \%$, cell transfection was performed with HDAC2 siRNA or control siRNA (Santa Cruz Biotechnology, Inc.) using Lipofectamine 2000 (Invitrogen; Thermo Fisher Scientific, Inc.) in ESCC EC9706 cells, according to the manufacturer's protocol.

Cell proliferation assay by Cell Counting Kit-8 (CCK-8). Cell proliferation was determined using Cell Counting Kit-8 (Beyotime Institute of Biotechnology, Haimen, China) according to the manufacturer's protocol. In brief, EC9706 cells that underwent different treatments (untreated, and transfected with control siRNA or HDAC2 siRNA) were seeded into 96-well culture plates $(2,000$ cells/well). When measured, $10 \%$ CCK- 8 was added to 96 -well culture plates, and the absorbance value at $450 \mathrm{~nm}$ was determined using a microplate reader. The experiment was repeated 3 times.

Cell cycle assay by flow cytometry. The cell cycle assay was performed as previously described (21). In brief, the three groups of EC9706 cells aforementioned were harvested (1x10 ${ }^{6}$ cells per group) $48 \mathrm{~h}$ subsequent to transfection, washed with PBS and fixed with $70 \%$ ethanol at $4^{\circ} \mathrm{C}$ for $30 \mathrm{~min}$. Subsequent to washing 3 more times with cold PBS, the cells were resuspended in PBS containing $40 \mu \mathrm{g}$ propidium iodide and $100 \mu \mathrm{g}$ RNase A, and incubated at $37^{\circ} \mathrm{C}$ for $30 \mathrm{~min}$. Finally, DNA content was analyzed by flow cytometry. This experiment was repeated 3 times.

Apoptosis detection by flow cytometry. Cell apoptosis detection was performed as previously described (22). Briefly, the three groups of EC9706 cells were harvested $48 \mathrm{~h}$ after transfection, washed with cold PBS, and resuspended to a concentration of $1 \times 10^{6} / \mathrm{ml} .100 \mu \mathrm{l}$ of the cells were then transferred to the flow tube, followed by $5 \mu \mathrm{l}$ Annexin V-FITC (BD Biosciences, Franklin Lakes, NJ, USA) and $5 \mu 1$ propidium iodide (PI) and incubated in the dark for $15 \mathrm{~min}$. Finally, $1 \times 10^{4}$ cells were tested and analyzed by CellQuest (BD Biosciences). The experiment was repeated 3 times.

Western blot analysis. Western blotting was performed as described in a previous study (21). The three groups of EC9706 cells were lysed $48 \mathrm{~h}$ subsequent to transfection. The total protein was extracted and analyzed by western blotting, according to the standard procedure. The total protein extract was separated by $10 \%$ SDS-PAGE, and transferred to nitrocellulose (NC) membrane. The NC membrane was blocked in TBST containing 5\% skim milk for $2 \mathrm{~h}$ following the addition of the appropriate mouse monoclonal primary antibody [HDAC2, 
Table I. HDAC2 protein expression in ESCC, atypical hyperplasia and normal esophageal mucosa epithelial tissues.

\begin{tabular}{lcccccr}
\hline & & \multicolumn{3}{c}{ HDAC2 protein } & & \\
\cline { 4 - 6 } Groups & Total, $\mathrm{n}$ &,$+ \mathrm{n}$ &,$- \mathrm{n}$ & Expression rate, $\%$ & $\chi^{2}$ & P-value \\
\hline ESCC tissues & 69 & 55 & 14 & 79.71 & 44.121 & $<0.001$ \\
Mucosa adjacent to cancer & 45 & 23 & 22 & 51.11 & & \\
Normal esophageal mucosa epithelial tissues & 69 & 16 & 53 & 23.19 & & \\
\hline
\end{tabular}

HDAC2, histone deacetylase 2; ESCC, esophageal squamous cell carcinoma; +, expressed; -, not expressed.

Table II. The association between HDAC2 protein expression and clinicopathological features in esophageal squamous cell carcinoma.

\begin{tabular}{|c|c|c|c|c|c|c|}
\hline \multirow{2}{*}{$\begin{array}{l}\text { Clinicopathological } \\
\text { features }\end{array}$} & \multirow[b]{2}{*}{ Total, $\mathrm{n}$} & \multicolumn{3}{|c|}{ HDAC2 protein } & \multirow[b]{2}{*}{$\chi^{2}$} & \multirow[b]{2}{*}{$\mathrm{P}$-value } \\
\hline & &,$+ \mathrm{n}$ &,$- \mathrm{n}$ & Expression rate, $\%$ & & \\
\hline Age & & & & & 0.086 & 0.769 \\
\hline$>60$ years & 42 & 33 & 9 & 78.57 & & \\
\hline$\leq 60$ years & 27 & 22 & 5 & 81.48 & & \\
\hline Gender & & & & & 1.050 & 0.305 \\
\hline Male & 41 & 31 & 10 & 75.61 & & \\
\hline Female & 28 & 24 & 4 & 85.71 & & \\
\hline Histological grade & & & & & 8.003 & 0.018 \\
\hline I & 19 & 11 & 8 & 57.89 & & \\
\hline II & 27 & 23 & 4 & 85.19 & & \\
\hline III & 23 & 21 & 2 & 91.30 & & \\
\hline Invasive depth & & & & & 8.490 & 0.004 \\
\hline Superficial layer & 22 & 13 & 9 & 59.09 & & \\
\hline Deep layer & 47 & 42 & 5 & 89.36 & & \\
\hline TNM staging & & & & & 6.654 & 0.010 \\
\hline $\mathrm{I}+\mathrm{II}$ & 33 & 22 & 11 & 66.67 & & \\
\hline $\mathrm{III+IV}$ & 36 & 33 & 3 & 91.67 & & \\
\hline Lymph node metastasis & & & & & 10.134 & 0.001 \\
\hline No & 38 & 25 & 13 & 65.79 & & \\
\hline Yes & 31 & 30 & 1 & 96.77 & & \\
\hline
\end{tabular}

HDAC2, histone deacetylase 2; TNM, tumor-node-metastasis; +, expressed; -, not expressed.

catalog no., sc-81599; cyclin D1, sc-70899; p21, sc-817; B cell lymphoma-2 (Bcl-2), sc-56015; Bcl-2-associated X protein (Bax), sc-70407; $\beta$-actin, sc-47778; all dilutions, 1:200] and incubated overnight at $4^{\circ} \mathrm{C}$. The membrane was then incubated with an appropriate secondary antibody at room temperature for $1.5 \mathrm{~h}$. The NC membrane was soaked in enhanced chemiluminescence reagent for 1-3 min. Specific protein bands were developed in a dark room. The protein expression level was analyzed by gray-value analysis using Image-Pro 5.0 software, with the relative expression level indicated by the ratio of target protein to the reference protein ( $\beta$-actin).

Statistical analysis. All data were analyzed using SPSS 13.0 software (SPSS, Inc., Chicago, IL, USA). $\chi^{2}$ test, t-test or one-way analysis of variance was used to compare the differences, as appropriate. The least significant difference test was used for post-hoc analysis. $\mathrm{P}<0.05$ was considered to indicate a statistically significant difference.

\section{Results}

Increased HDAC2 protein expression in ESCC tissues. To examine the expression and localization of HDAC2 in ESCC tissues, immunohistochemistry was performed on serial sections of ESCC, atypical hyperplasia and normal esophageal tissues. It was found that HDAC2 protein was localized in the nucleus of ESCC (Fig. 1), and expression of HDAC2 protein in ESCC $(79.71 \%$; 55/69) was significantly increased compared 

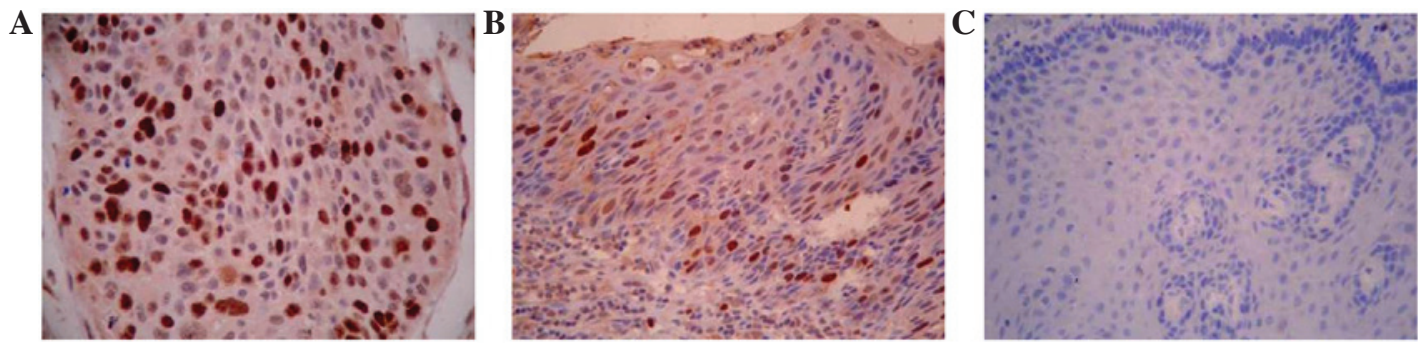

Figure 1. Immunohistochemical analysis for the expression of HDAC2 protein in ESCC tissues, atypical hyperplasia tissues and normal esophageal mucosa epithelial tissues. (A) Strong positive expression of HDAC2 protein in ESCC tissues; (B) positive expression of HDAC2 protein in atypical hyperplasia tissues; (C) negative expression of HDAC2 protein in normal esophageal mucosa epithelial tissues. HDAC2, histone deacetylase 2; ESCC, esophageal squamous cell carcinoma.

A

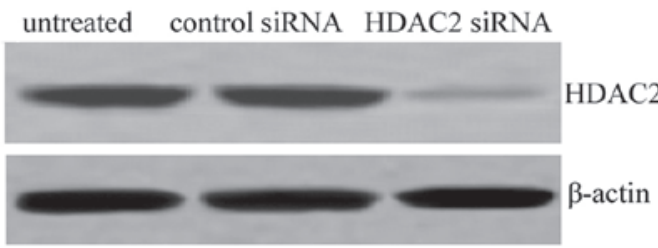

B

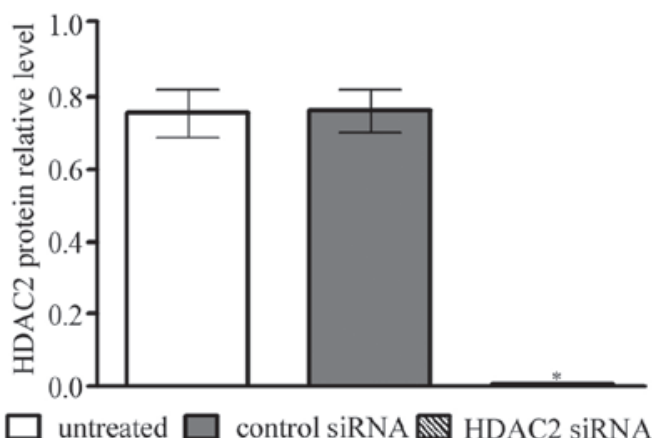

Figure 2. HDAC2 siRNA effectively downregulated HDAC2 protein expression in EC9706 cells. EC9706 cells were harvested $48 \mathrm{~h}$ subsequent to transfection with HDAC2 siRNA and control siRNA or non-treatment, and western blotting was utilized to detect the expression of HDAC2 protein. (A) Western blot analysis for HDAC2 protein expression in the untreated group, control siRNA group and HDAC2 siRNA group. (B) Semi-quantitative analysis of HDAC2 protein level in three groups of EC9706 cells. $\beta$-actin was used as an internal control. ${ }^{*} \mathrm{P}<0.05$ vs. untreated and control siRNA groups. HDAC2, histone deacetylase 2; siRNA, small interfering RNA.

with atypical hyperplasia tissues $(51.11 \% ; 23 / 45)$ and normal esophageal mucosa $(23.19 \% ; 16 / 69) \quad\left(\chi^{2}=44.121 ; \mathrm{P}<0.001\right)$ (Table I). These findings suggest that HDAC2 plays an important role in the occurrence and development of ESCC.

Association between HDAC2 expression and the clinicopathological features of patients with ESCC. To confirm the underlying role of HDAC2 expression in the occurrence and development of ESCC, the present study analyzed the association between the expression of HDAC2 protein and clinicopathological features, including age, gender, histological grade, invasive depth, TNM staging and lymph node metastasis. As shown in Table II, no significant associations were found between HDAC2 expression and age or gender (both $\mathrm{P}>0.05)$. By contrast, HDAC2 expression was associated with histological grade, invasive depth, TNM staging and lymph node metastasis (all $\mathrm{P}<0.05$; Table II), indicating that HDAC2 plays a pivotal role in the onset and development of ESCC.

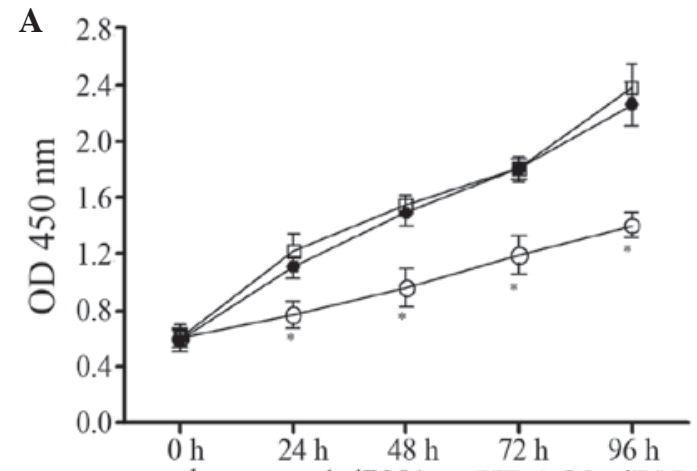

๑ untreated $\bullet$ control siRNA $\odot$ HDAC2 siRNA

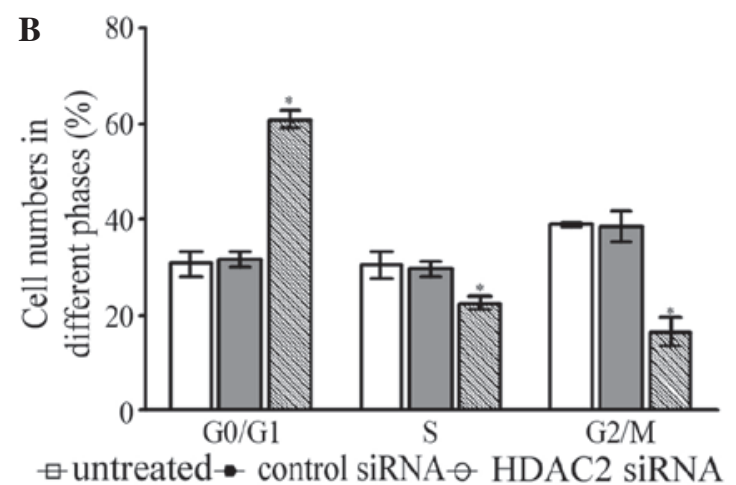

Figure 3. HDAC2 downregulation suppressed cell proliferation and altered cell cycle distribution in EC9706 cells. EC9706 cells were harvested $48 \mathrm{~h}$ subsequent to transfection with HDAC2 siRNA and control siRNA or non-treatment, and cell proliferation assay and cell cycle detection was performed. (A) HDAC2 downregulation suppressed cell proliferation in EC9706 cells. (B) Reduced HDAC2 level arrested the cell cycle in the G0/G1 phase in EC9706 cells, and cell cycle results derived from three independent experiments were expressed as the mean \pm standard deviation in EC9706 cells. ${ }^{*} \mathrm{P}<0.05$ vs. untreated and control siRNA groups. HDAC2, histone deacetylase 2; siRNA, small interfering RNA.

HDAC2 siRNA effectively reduced HDAC2 protein expression in EC9706 cells. To preliminarily elucidate the function of HDAC2 in ESCC, HDAC2 siRNA was transfected into EC9706 cells, and western blotting was performed to determine the HDAC2 protein expression $48 \mathrm{~h}$ after transfection. The results showed that compared with the untreated group and control siRNA group, HDAC2 protein expression was significantly decreased $(\mathrm{P}<0.001)$ (Fig. 2) in the HDAC2 siRNA group, indicating that HDAC2 siRNA significantly downregulated HDAC2 protein expression in EC9706 cells. 
A

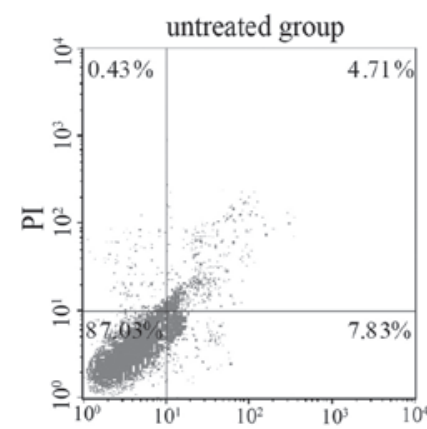

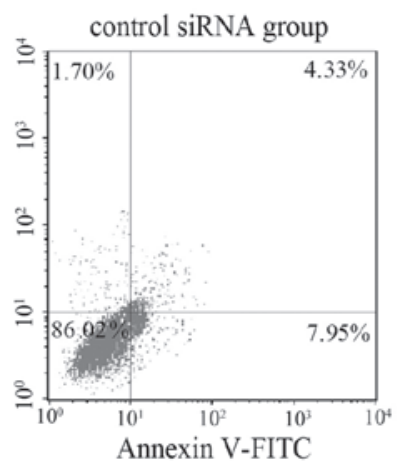

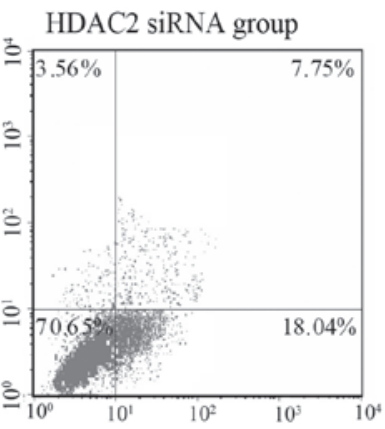

B

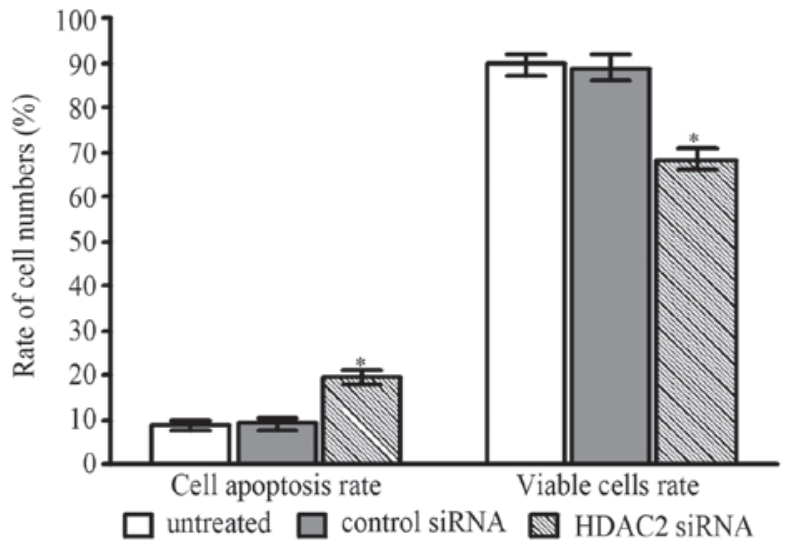

Figure 4. Effect of HDAC2 downregulation on cell apoptosis in EC9706 cells. EC9706 cells were harvested 48 h subsequent to transfection with HDAC2 siRNA and control siRNA or non-treatment, and cell apoptosis was detected by flow cytometry. (A) HDAC2 depletion induced apoptosis in EC9706 cells. (B) Cell apoptosis results derived from three independent experiments were expressed as the mean \pm standard deviation in EC 9706 cells. $\mathrm{P}<0.05$ vs. untreated and control siRNA groups. HDAC2, histone deacetylase 2; siRNA, small interfering RNA; FITC, fluorescein isothiocyanate.

A

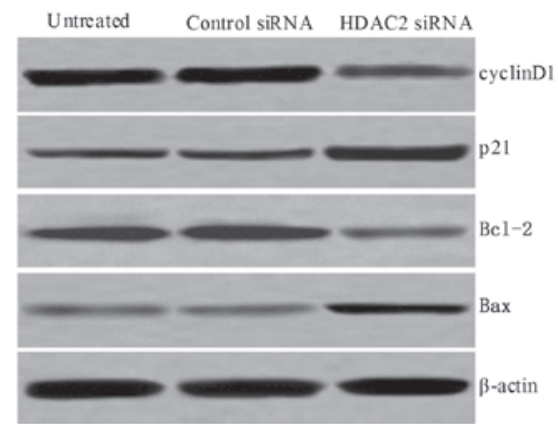

B

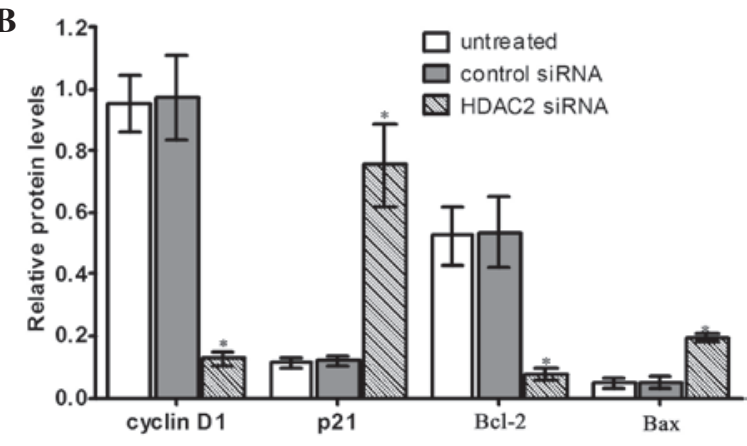

Figure 5. Effect of HDAC2 downregulation on the expression of cyclin D1, p21, Bcl-2 and Bax proteins in EC9706 cells. EC9706 cells were harvested $48 \mathrm{~h}$ subsequent to transfection with HDAC2 siRNA and control siRNA or non-treatment, and the expression of proteins was detected by western blot analysis. (A) HDAC2 downregulation decreased cyclin D1 and Bcl-2 protein expression and increased $\mathrm{p} 21$ and Bax protein expression. (B) The relative expression level of the proteins was assessed according to the ratio of the level of the interest protein in comparison to the $\beta$-actin level. ${ }^{*} \mathrm{P}<0.05$ vs. untreated and control siRNA groups. HDAC2, histone deacetylase 2; siRNA, small interfering RNA; Bcl-2, B cell lymphoma 2; Bax, Bcl-2-associated X protein.
HDAC2 downregulation inhibited cell proliferation and altered cell cycle distribution in EC9706 cells. To assess the biological role of HDAC2 in ESCC cells, the effects of HDAC2 downregulation on cell proliferation and cell cycle were investigated in EC9706 cells. The results revealed that HDAC2 siRNA inhibited proliferation of EC9706 cells compared with the untreated group and control siRNA group $(\mathrm{P}<0.001)$ (Fig. 3A). In addition, the HDAC2 siRNA group contained a significantly increased proportion of $\mathrm{G} 0 / \mathrm{G} 1$ phase cells $(60.95 \pm 1.92 \%)$ compared with the untreated group $(30.80 \pm 2.68 \%)$ and control siRNA group $(31.66 \pm 1.58 \%)$ $(\mathrm{F}=198.794 ; \mathrm{P}<0.001)$ (Fig. 3B). Subsequently, it was found that the HDAC2 siRNA group contained a significantly decreased proportion of S phase cells $(22.58 \pm 1.22 \%)$ compared with the untreated group $(30.40 \pm 2.90 \%)$ and control siRNA group $(29.61 \pm 1.66 \%)(\mathrm{F}=13.215 ; \mathrm{P}=0.006)$ (Fig. 3B). These data suggest that HDAC2 plays an essential role in the regulation of cell proliferation and cell cycle progression in ESCC.

Suppression of HDAC2 expression induced cell apoptosis in EC9706 cells. To explore whether HDAC2 downregulation affects apoptosis in EC9706 cells, flow cytometry was utilized to investigate cell apoptosis in EC9706 cells subsequent to various treatments. The results demonstrated that the number of apoptotic cells in HDAC2 siRNA group $(19.57 \pm 1.53 \%)$ was significantly increased compared with the untreated group $(8.85 \pm 1.02 \%)$ and control siRNA group $(9.13 \pm 1.18 \%)(\mathrm{F}=70.383$; $\mathrm{P}<0.001$; Fig. $4 \mathrm{~A}$ and $\mathrm{B})$. In addition, the HDAC2 siRNA group exhibited a significantly decreased number of viable cells 
(68.33 $\pm 2.32 \%)$ compared with the untreated group $(89.65 \pm 2.62 \%)$ and control siRNA group $(88.99 \pm 2.97 \%)(\mathrm{F}=62.784 ; \mathrm{P}<0.001)$ (Fig. 4A and B). These findings indicate that HDAC2 downregulation markedly induces cell apoptosis in EC9706 cells.

Cell proliferation, cell cycle and apoptosis-associated protein assays. To preliminarily clarify the possible molecular mechanisms of proliferation inhibition, cell cycle alteration and apoptosis induction mediated by HDAC2 downregulation, western blotting was utilized to investigate the expression of cell proliferation, cell cycle, and apoptosis-associated proteins, including cyclin D1, p21, Bcl-2 and Bax. The results showed that HDAC2 siRNA significantly reduced the expression of cyclin D1 and Bcl-2, but increased the expression of p21 and Bax, compared with the untreated group and control siRNA group $(\mathrm{P}<0.001)$ (Fig. 5). These findings indicate that HDAC2 downregulation mediated proliferation inhibition, alteration of cell cycle distribution and cell apoptosis are associated with decrease in cyclin D1 and Bcl-2 protein expression and increase in $\mathrm{p} 21$ and Bax protein expression.

\section{Discussion}

Studies show that HDAC2 expression, a member of the deacetylase family, is associated with tumor onset and development $(23,24)$. A high level of HDAC2 is an independent prognostic factor for various types of tumors, including oral, ovarian, endometrial and gastric cancers $(6,10,15)$. Fritzsche et al (25) reported a 60\% increase in HDAC2 expression in renal cancer. Adams et al found that HDAC2 was also highly expressed in Hodgkin's lymphoma (26). HDAC2 expression was significantly increased in endometrial carcinoma compared with normal endometrial tissue (27). Therefore, the present study investigated HDAC2 expression in ESCC, adjacent atypical hyperplasia and normal esophageal tissues by immunohistochemistry. The results showed that 55 out of 69 cases of ESCC showed HDAC2expression (79.71\%), significantly increased compared with adjacent atypical hyperplasia (51.11\%) and normal esophageal mucosa (23.19\%), suggesting that HDAC2 may play an important role in the onset and development of ESCC. However, the molecular mechanism requires elucidation. To confirm the possible role of HDAC2 downregulation in ESCC, we transfected HDAC2 siRNA in ESCC EC9706 cells. Expression of HDAC2 was verified by western blot analysis. The results confirmed that HDAC2 siRNA effectively downregulated HDAC2 expression in EC9706 cells, providing a basis for further functional studies.

Previous studies found that HDAC2 played an important role in tumor cell proliferation, cell cycle control and other cellular processes $(28,29)$. A high level of HDAC expression promotes tumoral cell proliferation, regulates the cell cycle, increases tumor angiogenesis, and upregulates the expression of various oncogenes (27). HDAC is overexpressed in numerous types of tumors, coupled with inhibition of tumor suppressor gene expression and accelerations of tumor cell proliferation. HDAC2 specifically binds to the promoter of $\mathrm{p} 21^{\mathrm{WAF} / \mathrm{CIP} 1}$ and thus inhibits p21 expression, thereby promoting cell cycle progression (30). Previous studies have shown that overexpression of HDACs is closely correlated with decreased expression of p21 (14,31), an important inhibitor of cyclin-dependent kinases, and therefore promotes rapid proliferation of the cells. In addition, suppression of HDAC2 expression upregulates p21 expression, and downregulates expression of cyclin D1 and cyclin A (27), which impedes cell cycle progression. To further understand whether downregulation of HDAC2 in EC9706 regulated cell proliferation and cell cycle progression, changes in cell proliferation and cell cycle progression in different treatment groups of EC9706 cells were detected. The present findings demonstrated that suppression of HDAC2 significantly inhibited proliferation of EC9706 cells. Stepwise investigation showed that the HDAC2 siRNA group contained a significantly increased proportion of G0/G1 phase cells $(\mathrm{P}<0.001)$ and significantly decreased proportion of $\mathrm{S}$ phase cells $(\mathrm{P}=0.006)$ compared with the untreated group and control siRNA group, indicating that HDAC2 overexpression induced cell cycle arrest at the G0/G1 phase and reduced the percentage of $\mathrm{S}$ phase cells. This resulted in the blockage of DNA synthesis and cell division, and thus cell proliferation was suppressed. To further elucidate the underlying molecular mechanism, cell cycle-associated cyclin D1 and p21 were examined. The results showed that HDAC2 suppression upregulated p21 expression and downregulated cyclin D1 expression, indicating that DHAC2 suppression-induced inhibition of proliferation and cell cycle arrest may be associated with changes in expression of cyclin D1 and p21.

Currently, a plethora of evidence supports the anti-apoptotic effect of HDAC2 in tumor cells. Knockdown of HDAC2 in tumor cells leads to terminally differentiated phenotypes and p21 upregulation-mediated apoptosis. Knockdown of HDAC2 in breast cancer cells may induce the binding activity of p53, resulting in proliferation inhibition and cellular senescence (32). Inhibition of HDAC2 by histone deacetylase inhibitors reduced the expression level of the anti-apoptotic gene Bcl-2, and thus inducing apoptosis (33). In the present study, it was found that suppression of HDAC2 significantly induced apoptosis in EC9706 cells. The present study further analyzed the expression of two key apoptosis-associated proteins (anti-apoptotic Bcl-2 and pro-apoptotic Bax). The results showed that HDAC2 suppression induced downregulation of Bcl-2 and upregulation of Bax, suggesting that HDAC2 suppression-induced apoptosis of ESCC cells may be associated with downregulation of Bcl-2 and upregulation of Bax. However, the exact molecular mechanism remains to be elucidated.

In conclusion, the present results suggest that HDAC2 overexpression is closely associated with the onset and progression of ESCC. Suppression of HDAC2 expression inhibited proliferation, induced cell cycle arrest, and induced cell apoptosis in EC9706 cells. These changes may be associated with upregulation of cyclin D1 and Bcl-2, and downregulation of p21 and Bax. Additional study of the HDAC2 function may provide a theoretical basis for target therapy of the patients with ESCC.

\section{Acknowledgements}

This study was supported by the National Natural Science Foundation of China (grant no. 81372677).

\section{References}

1. Parkin DM, Bray F, Ferlay J and Pisani P: Global cancer statistics, 2002. CA Cancer J Clin 55: 74-108, 2005. 
2. Lam AK: Molecular biology of esophageal squamous cell carcinoma. Crit Rev Oncol Hematol 33: 71-90, 2000.

3. Parkin DM, Bray FI and Devesa SS: Cancer burden in the year 2000. The global picture. Eur J Cancer 37 (Suppl 8): S4-S66, 2001.

4. Ekman S, Dreilich M, Lennartsson J, Wallner B, Brattström D, Sundbom M and Bergqvist M: Esophageal cancer: Current and emerging therapy modalities. Expert Rev Anticancer Ther 8: 1433-1448, 2008.

5. Ke L: Mortality and incidence trends from esophagus cancer in selected geographic areas of China circa 1970-90. Int J Cancer 102: 271-274, 2002.

6. Edwards BK, Brown ML, Wingo PA, Howe HL, Ward E, Ries LA, Schrag D, Jamison PM, Jemal A, Wu XC, et al: Annual report to the nation on the status of cancer, 1975-2002, featuring population-based trends in cancer treatment. J Natl Cancer Inst 97: 1407-1427, 2005.

7. Brandl A, Heinzel $\mathrm{T}$ and Krämer $\mathrm{OH}$ : Histone deacetylases: Salesmen and customers in the post-translational modification market. Biol Cell 101: 193-205, 2009.

8. Krämer OH: HDAC2: A critical factor in health and disease. Trends Pharmacol Sci 30: 647-655, 2009.

9. Wagner $\mathrm{T}$, Brand $\mathrm{P}$, Heinzel $\mathrm{T}$ and Krämer $\mathrm{OH}$ : Histone deacetylase 2 controls p53 and is a critical factor in tumorigenesis. Biochim Biophys Acta 1846: 524-538, 2014.

10. Bieliauskas AV and Pflum MK: Isoform-selective histone deacetylase inhibitors. Chem Soc Rev 37: 1402-1413, 2008.

11. Weichert W: HDAC expression and clinical prognosis in human malignancies. Cancer Lett 280: 168-176, 2009

12. Chang HH, Chiang CP, Hung HC, Lin CY, Deng YT and Kuo MY: Histone deacetylase 2 expression predicts poorer prognosis in oral cancer patients. Oral Oncol 45: 610-614, 2009.

13. Zhu P, Martin E, Mengwasser J, Schlag P, Janssen KP and Göttlicher M: Induction of HDAC2 expression upon loss of APC in colorectal tumorigenesis. Cancer Cell 5: 455-463, 2004.

14. Huang BH, Laban M, Leung CH, Lee L, Lee CK, Salto-Tellez M, Raju GC and Hooi SC: Inhibition of histone deacetylase 2 increases apoptosis and p21Cip1/WAF1 expression, independent of histone deacetylase 1. Cell Death Differ 12: 395-404, 2005.

15. Ye F, Chen Y, Hoang T, Montgomery RL, Zhao XH, Bu H, Hu T, Taketo MM, van Es JH, Clevers H, et al: HDAC1 and HDAC2 regulate oligodendrocyte differentiation by disrupting the beta-catenin-TCF interaction. Nat Neurosci 12: 829-838, 2009.

16. Fritsche P, Seidler B, Schüler S, Schnieke A, Göttlicher M, Schmid RM, Saur D and Schneider G: HDAC2 mediates therapeutic resistance of pancreatic cancer cells via the BH3-only protein NOXA. Gut 58: 1399-1409, 2009.

17. Bug G, Ritter M, Wassmann B, Schoch C, Heinzel T, Schwarz K, Romanski A, Kramer OH, Kampfmann M, Hoelzer D, et al: Clinical trial of valproic acid and all-trans retinoic acid in patients with poor-risk acute myeloid leukemia. Cancer 104: 2717-2725, 2005.

18. Munster PN, Marchion D, Thomas S, Egorin M, Minton S, Springett G, Lee JH, Simon G, Chiappori A, Sullivan D and Daud A: Phase I trial of vorinostat and doxorubicin in solid tumours: Histone deacetylase 2 expression as a predictive marker. Br J Cancer 101: 1044-1050, 2009.

19. Li B, Guo Q, Yang H, Guan J, Liu Z, Li S, Liu G and Wei L: Left thoracoabdominal approach in surgical treatment of adenocarcinoma of the esophagogastric junction in the Northern Henan Province of China. Dig Surg28: 2-8, 2011.
20. Liu HT, Wang N, Wang X and Li SL: Overexpression of Pim-1 is associated with poor prognosis in patients with esophageal squamous cell carcinoma. J Surg Oncol 102: 683-688, 2010.

21. Lu Z, Liu H, Xue L, Xu P, Gong T and Hou G: An activated Notchl signaling pathway inhibits cell proliferation and induces apoptosis in human esophageal squamous cell carcinoma cell line EC9706. Int J Oncol 32: 643-651, 2008.

22. Yan L, Li S, Xu C, Zhao X, Hao B, Li H and Qiao B: Target protein for Xklp2 (TPX2), a microtubule-related protein, contributes to malignant phenotype in bladder carcinoma. Tumour Biol 34: 4089-4100, 2013.

23. Ye P, Xing H, Lou F, Wang K, Pan Q, Zhou X, Gong L and Li D: Histone deacetylase 2 regulates doxorubicin (Dox) sensitivity of colorectal cancer cells by targeting ABCB1 transcription. Cancer Chemother Pharmacol 77: 613-621, 2016.

24. Ler SY, Leung CH, Khin LW, Lu GD, Salto-Tellez M, Hartman M, Iau PT, Yap CT and Hooi SC: HDAC1 and HDAC2 independently predict mortality in hepatocellular carcinoma by a competing risk regression model in a Southeast Asian population. Oncol Rep 34: 2238-2250, 2015.

25. Fritzsche FR, Weichert W, Röske A, Gekeler V, Beckers T, Stephan C, Jung K, Scholman K, Denkert C, Dietel M and Kristiansen G: Class I histone deacetylases 1,2 and 3 are highly expressed in renal cell cancer. BMC Cancer 8: 381, 2008.

26. Adams H, Fritzsche FR, Dirnhofer S, Kristiansen G and Tzankov A: Class I histone deacetylases 1,2 and 3 are highly expressed in classical Hodgkin's lymphoma. Expert Opin Ther Targets 14: 577-584, 2010

27. Fakhry H, Miyamoto T, Kashima H, Suzuki A, Ke H, Konishi I and Shiozawa T: Immunohistochemical detection of histone deacetylases in endometrial carcinoma: Involvement of histone deacetylase 2 in the proliferation of endometrial carcinoma cells. Hum Pathol 41: 848-858, 2010.

28. Zhu P, Huber E, Kiefer F and Göttlicher M: Specific and redundant functions of histone deacetylases in regulation of cell cycle and apoptosis. Cell Cycle 3: 1240-1242, 2004.

29. Jurkin J, Zupkovitz G, Lagger S, Grausenburger R, Hagelkruys A, Kenner $L$ and Seiser C: Distinct and redundant functions of histone deacetylases HDAC1 and HDAC2 in proliferation and tumorigenesis. Cell Cycle 10: 406-412, 2011.

30. Yamaguchi T, Cubizolles F, Zhang Y, Reichert N, Kohler H, Seiser $C$ and Matthias P: Histone deacetylases 1 and 2 act in concert to promote the G1-to-S progression. Genes Dev 24: 455-469, 2010

31. Hrzenjak A, Moinfar F, Kremser ML, Strohmeier B, Staber PB, Zatloukal K and Denk H: Valproate inhibition of histone deacetylase 2 affects differentiation and decreases proliferation of endometrial stromal sarcoma cells. Mol Cancer Ther 5: 2203-2210, 2006

32. Harms KL and Chen X: Histone deacetylase 2 modulates p53 transcriptional activities through regulation of p53-DNA binding activity. Cancer Res 67: 3145-3152, 2007.

33. Duan H, Heckman CA and Boxer LM: Histone deacetylase inhibitors down-regulate bcl-2 expression and induce apoptosis in $\mathrm{t}(14 ; 18)$ lymphomas. Mol Cell Biol 25: 1608-1619, 2005. 